\title{
Miembros del Comité Editorial Asesor de la Revista Médica de Chile, desde 1973 hasta 2004
}

\author{
Members of the Editorial Advisory \\ Committee of Revista Médica de \\ Chile, from 1973 to 2004
}

Since the late 1960's, Revista Médica de Chile requests external peer reviewers to assess manuscripts received. In 1973, a group of distinguished participants were selected to form the Editorial Advisory Committee that has been changed four times since then. As a token of appreciation for their important help in the editorial process, the names of all who have been part of this Committee are reproduced below (Rev Méd Chile 2005; 133: 129-31).

(Key Words: Journalism, medical; Manuscripts, medical; Peer Review)

$\mathrm{U}$ n hito en el progreso de la Revista Médica de Chile fue institucionalizar la revisión crítica de los manuscritos por especialistas externos, previo a la toma de decisiones por los editores. Iniciada a fines de la década de 1960, su amplitud ha crecido progresivamente. En el año calendario 2003 intervinieron 342 personas, muchas de las cuales revisaron más de un manuscrito en ese año.

Desde 1973, la Revista ha nominado a destacados revisores externos como miembros estables de su Comité Editorial Asesor. Ellos actúan como revisores externos y, además, asesoran a los editores en casos de divergencias de opiniones entre los especialistas consultados. Su labor, generosa y voluntaria, ha contribuido al perfeccionamiento de los manuscritos antes de ser publicados y ha ayudado a los editores en la adopción de decisiones que son de su responsabilidad final.

Coincidiendo con un nuevo cambio en la nómina de miembros de este Comité, se reproducen en este artículo las nóminas de quienes han sido sus miembros, desde 1973 hasta 2004. Este es un homenaje a personas que han dado su tiempo, voluntad y experiencia para contribuir en una obra que enorgullece a las instituciones beneficiadas y a la medicina nacional. 
1973-1977

Mordo Alvo G

Antonio Arteaga Ll

Pablo Casanegra $\mathrm{P}$

Edgardo Carrasco C

Gastón Chamorro S

Gastón Chamorro Z

Edgardo Escobar $\mathrm{C}$

Rafael del Río de la Torre

Germán Ducach G

Ricardo Ferretti D

Ramón Florenzano G

Arnaldo Foradori $\mathrm{C}$

Eduardo Katz C

Ricardo Katz U

Camilo Larraín A

Manuel Losada L

Ernesto Medina L

Ernesto Oberhauser A

José Manuel Orellana Alcalde

Tulio Pizzi P

Renato Palma C

Hugo Pumarino C

Mario Plaza de los Reyes T

Manuel Rodríguez L

Fernando Rufín D

Mario Salcedo S

Guillermo Ugarte M

Luis Vargas Fernández

Marta Velasco R

Fernando Valenzuela $\mathrm{R}$

\section{8-1983 (Junio)}

Rodolfo Armas Merino

Mario Andreis C

Roberto Arinoviche Sch

Antonio Arteaga Ll

Werner Apt B

Francisco Beas $\mathrm{F}$

Cristián Bianchi $\mathrm{C}$

Gastón Chamorro S

Alberto Daiber E

Hugo Donoso P

Ricardo Ferreti D

Ramón Florenzano G

Gerardo Gómez E

Hernán Iturriaga $\mathrm{R}$
Jaime Klinger $\mathrm{R}$

Camilo Larraín A

Enrique López Caffarena

Ernesto Medina L

Carlos Muñoz A

Renato Palma C

Leonardo Paredes $\mathrm{P}$

Esteban Parrochia B

Jaime Pérez Olea

Gustavo Pineda V

Hernán Reyes M

Emilio Roessler B

Oscar Román A

Guillermo Ugarte M

José Manuel Ugarte A

Gloria Valdés S

Vicente Valdivieso D

Luis Vargas Fernández

Odette Veit A

1983 (Julio) - 1996 (Noviembre)

Juan Pablo Allamand G

Werner Apt B

Aquiles Arancibia $\mathrm{O}$

Rodolfo Armas Merino

Antonio Arteaga Ll

Aurelio Carvallo V

Gastón Chamorro S

Hugo Donoso P

Ricardo Ferreti D

Ramón Florenzano U

Manuel García de los Ríos A

Patricio González G

Pedro Hoffenberg F

Hernán Iturriaga $R$

Sergio Jacobelli G

Ana María Kaempffer R

Camilo Larraín A

Osvaldo Llanos L

Enrique López Caffarena

Jorge Meruane S

Ernesto Medina L

Jorge Morales B

Leonardo Paredes $\mathrm{P}$

Jaime Pérez Olea

Jorge Pfau D

Gustavo Pineda V 
Valeria Prado J

Hugo Pumarino C

Hernán Reyes M

Manuel Rodríguez L

Emilio Roessler B

Oscar Román A

Eduardo Rosselot J

Guillermo Ugarte M

Gloria Valdés S

Vicente Valdivieso D

Luis Vargas Fernández

Odette Veit A

Gabriela Venturini R

1996 (Diciembre) - 2004

Guillermo Acuña L

Gonzalo Alvarez U

Patricia Ancic C

Isabel Avendaño B

Arturo Ayala B

José Manuel Borgoño D

Ramón Corbalán $\mathrm{H}$

Raúl Domenech L

Susana Elgueta M

Enrique Gallardo I

Juan Giaconi G

Sergio Gloger K

Jorge Gutiérrez C
Leonardo Guzmán B

Pedro Herskovic L

Juan Pablo García Huidobro-Toro

Chrystal Juliet L

Claudio Liberman G

Pablo Lira V

Carmen Lisboa B

Fernando Lolas S

José Manuel López M

Pedro Maggiolo G

Iris Mella G

Antonio Morales B

Félix Muñoz C

Flavio Nervi O

Manuel Oyarzún G

Miguel Oyonarte G

Santiago Rivero D

Manuel Santos A

Cecilia Sepúlveda C

Juan Jorge Silva S

Eugenio Spencer $\mathrm{O}$

Sergio Thambo B

Ricardo Uauy D

Andrés Valdivieso D

Fernando Vergara E

Nicolás Velasco $\mathrm{F}$

Juan Carlos Weitz V 